\title{
Submitted: 1 Axillary adenopathy after COVID-19 vaccine Accepted: in patients undergoing breast ultrasound
} 05.11.2021

Published: 29.11 .2021

Keywords

ultrasound;

breast;

COVID-19;

lymphadenopathy; vaccine

\author{
Sara De Giorgis ${ }^{1}$, Alessandro Garlaschi², Nicole Brunetti', Simona Tosto², \\ Giuseppe Rescinito², Francesco Monetti2 , Claudio Oddone², Barbara \\ Massa $^{3}$, Francesca Pitto ${ }^{3}$, Massimo Calabrese ${ }^{2}$, Alberto Stefano Tagliafico ${ }^{1,2}$ \\ ${ }^{\prime}$ Radiology Section, Department of Health Sciences (DISSAL), University of Genova, Via L.B. \\ Alberti 2, 16132 Genoa, Italy \\ 2 Department of Radiology, IRCCS - Ospedale Policlinico San Martino, Largo Rosanna Benzi \\ 10, 16132 Genoa, Italy \\ ${ }^{3}$ Cyto-Histopathological Unit, IRCCS - Ospedale Policlinico San Martino, Largo Rosanna \\ Benzi 10, 16132 Genoa, Italy
}

Correspondence: Sara De Giorgis, Radiology Section, Department of Health Sciences (DISSAL), University of Genova, Via L.B. Alberti 2, 16132 Genoa, Italy; email: degiorgis.sara1990@gmail.com

DOI: $10.15557 / J o U .2021 .0060$

\begin{abstract}
After COVID-19 vaccination, a spectrum of axillary lymphadenopathy were observed in patients undergoing routine breast ultrasound. Malignancy remains the most serious differential in cases of unilateral axillary adenopathy. Knowledge of axillary ultrasound findings after COVID-19 vaccination is essential to prevent unnecessary biopsy or change in therapy in oncological patients. From March to May 2021, 10 female patients underwent breast ultrasound in our Department for the evaluation of axillary lumps. All the patients received their first or second dose of COVID-19 vaccine 20-30 days before the exam in the same extremity of the ultrasound evaluation where lymphadenopathy was found. Five patients had a personal history of previous breast cancer, and the radiologist decided to perform a core biopsy (the histology was negative for malignancy). The other five patients with no personal history of cancer underwent ultrasound and returned after a short-term follow-up. Regression of the enlarged lymph nodes was found.
\end{abstract}

\section{Introduction}

Italy was the first European country facing a COVID-19 outbreak, with northern regions being particularly severely affected by the pandemic ${ }^{(1)}$. Various methods are available to deal with the dramatic effects of the virus, but the development of a specific vaccine is considered the most effective way to control the spread of COVID-19. Since December 2020, several vaccines with $\mathrm{mRNA}$, vector, and protein subunit mechanisms have been developed and produced. Pfizer-BioNTech and Moderna were among the first vaccines approved for emergency use authorization (EUA) from the United States Food and Drug Administration (FDA) ${ }^{(2-4)}$. In Italy, the COVID-19 vaccination campaign started on 27 December 2020, when Italy received 9,750 doses of the Pfizer-BioNTech COVID-19 vaccine. The doses were entirely used over the following days to vaccinate part of the medical and health personnel of hospitals, just like in most countries in the European Union. As of 22 May 2021, Italy reached 30 million administered vaccine doses ["Vaccini, in Italia superata quota 30 milioni di dosi somministrate", tgcom24.mediaset.it. 22 May 2021, retrieved 22 May 2021], with 9.9 million people vaccinated with a double dose. Lymphadenopathy was reported for $0.3 \%$ and $1.1 \%$ of Pfizer-BioNTech and Moderna vaccines, respectively ${ }^{(5)}$. Few case studies on lymphadenopathy associated with COVID-19 vaccination have been published to date, especially in patients undergoing breast evaluation with ultrasound ${ }^{(6-10)}$. With the extensive rollout of the COVID-19 vaccines, radiologists should consider vaccine-induced adenopathy in the wide spectrum of differential diagnosis of adenopathy seen on breast imaging, especially in patients with a previous history of malignancy or breast cancer. 
Tab. 1. Breast ultrasound features of axillary lymph nodes

\begin{tabular}{|c|c|c|c|c|c|c|c|c|c|c|c|}
\hline 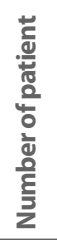 & 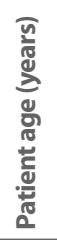 & 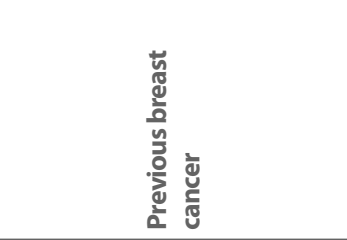 & 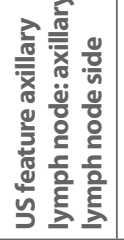 & 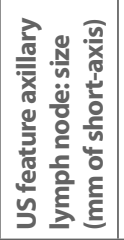 & 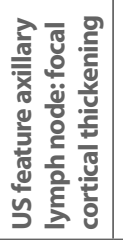 & 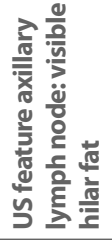 & 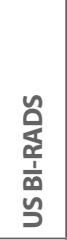 & 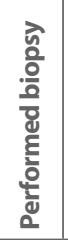 & 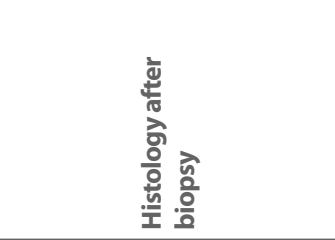 & 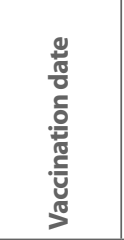 & 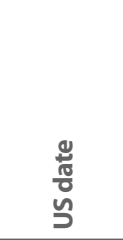 \\
\hline 1 & 29 & No & Left & 18 & No & Yes & E3 & No & No & $02 / 2021$ & $03 / 2021$ \\
\hline 2 & 66 & $\begin{array}{c}\text { Yes: in } 2017 \text { IDC in left breast } \\
\text { with negative ipsilateral } \\
\text { axillary lymph nodes }\end{array}$ & Left & 22 & No & Yes & E4b & Yes & $\begin{array}{c}\text { normal lymphocytes } \\
\text { and histiocytes in } \\
\text { hematoxylin-eosin staining }\end{array}$ & $02 / 2021$ & $03 / 2021$ \\
\hline 3 & 34 & No & Right & 11 & Yes & Yes & E3 & No & & $02 / 2021$ & $03 / 2021$ \\
\hline 4 & 54 & $\begin{array}{c}\text { Yes: in } 2015 \text { DCIS in left } \\
\text { breast with negative } \\
\text { ipsilateral axillary lymph } \\
\text { nodes }\end{array}$ & Left & 23 & No & Yes & $\mathrm{E} 4 \mathrm{~b}$ & Yes & $\begin{array}{l}\text { normal lymphocytes } \\
\text { and histiocytes in } \\
\text { hematoxylin-eosin staining }\end{array}$ & $03 / 2021$ & $04 / 2021$ \\
\hline 5 & 74 & No & Left & 15 & Yes & Yes & E3 & No & & $03 / 2021$ & $04 / 2021$ \\
\hline 6 & 46 & $\begin{array}{c}\text { Yes: in } 2018 \text { IDC in left breast } \\
\text { with negative ipsilateral } \\
\text { axillary lymph nodes }\end{array}$ & Left & 13 & Yes & Yes & E4b & Yes & $\begin{array}{c}\text { normal lymphocytes } \\
\text { and histiocytes in } \\
\text { hematoxylin-eosin staining }\end{array}$ & $03 / 2021$ & $05 / 2021$ \\
\hline 7 & 76 & $\begin{array}{c}\text { Yes: in } 2018 \text { IDC in right } \\
\text { breast with negative } \\
\text { ipsilateral axillary lymph } \\
\text { nodes }\end{array}$ & Right & 20 & No & Yes & $E 4 b$ & Yes & $\begin{array}{l}\text { normal lymphocytes } \\
\text { and histiocytes in } \\
\text { hematoxylin-eosin staining }\end{array}$ & $04 / 2021$ & $05 / 2021$ \\
\hline 8 & 59 & $\begin{array}{c}\text { Yes: in } 2020 \text { IDC in left breast } \\
\text { with negative ipsilateral } \\
\text { axillary lymph nodes }\end{array}$ & Left & 20 & No & Yes & $\mathrm{E} 4 \mathrm{~b}$ & Yes & $\begin{array}{l}\text { normal lymphocytes } \\
\text { and histiocytes in } \\
\text { hematoxylin-eosin staining }\end{array}$ & $04 / 2021$ & $05 / 2021$ \\
\hline 9 & 39 & No & Left & 22 & No & Yes & E3 & No & & $05 / 2021$ & $05 / 20121$ \\
\hline 10 & 28 & No & Right & 20 & Yes & Yes & E3 & No & & $05 / 2021$ & $05 / 2021$ \\
\hline
\end{tabular}

Here, we report axillary lymph node ultrasound findings after the administration of COVID-19 vaccine in patients undergoing routine breast imaging, with the aim to increase the awareness of relevant US features among radiologists. We also suggest a short-term follow-up for unilateral axillary adenopathy in the setting of recent COVID-19 vaccination to avoid immediate and unnecessary biopsies.

\section{Material and method}

From March to May 2021, 10 female patients (age range: 29-76 years) underwent breast ultrasound in our Department for the evaluation of palpable or nonpalpable axillary lumps found during routine breast imaging or during clinical examination (Tab. 1). All the patients were included in IRB approved clinical trials (NCT03033030, NCT02066142 and NCT04253366; CER $262 / 2019 ; 514 / 2014)$ and gave their informed consent.

All the patients received the first or second dose of a COVID-19 vaccine (BNT162b2 mRNA, BioNTech and Pfizer) 20-30 days before the exam in the same extremity of the ultrasound evaluation where lymphadenopathy was found. Axillary ultrasound showed enlarged axillary lymph nodes, and in four patients uniformly thickened cortices. In only one patient abnormal lymph nodes were present in the contralateral axilla.

\section{Results}

Five patients had a personal history of previous breast cancer, and the breast radiologist decided to perform a core biopsy (Breast Imaging Reporting and Data System BI-RADS 4b was assigned; BI-RADS 4 suggests a moderate suspicion for malignancy: $>10 \%$ to $<50 \%)$. The histology was negative for malignancy (Fig. 1).

The other five patients had no history of cancer. In cases of negative personal history of breast cancer, the radiologist assigned BIRADS 3 (a finding placed in this BI-RADS category should have less than $2 \%$ risk of malignancy) and suggested that patients should return for a short-term follow-up targeted ultrasound (after 4-8 week) of the axilla. The follow-up demonstrated regression of enlarged lymph nodes in these patients (Fig. 2).

BI-RADS system relates to the lesion in the breast: we decided to use this system in the study of lymphadenopathy in these patients to underline the importance of returning for a follow-up or to undergo a biopsy.

\section{Discussion}

The most common side effects associated with COVID19 vaccines include local injection site pain, fever, chills, myalgias, headache, and fatigue, which usually resolve in a 

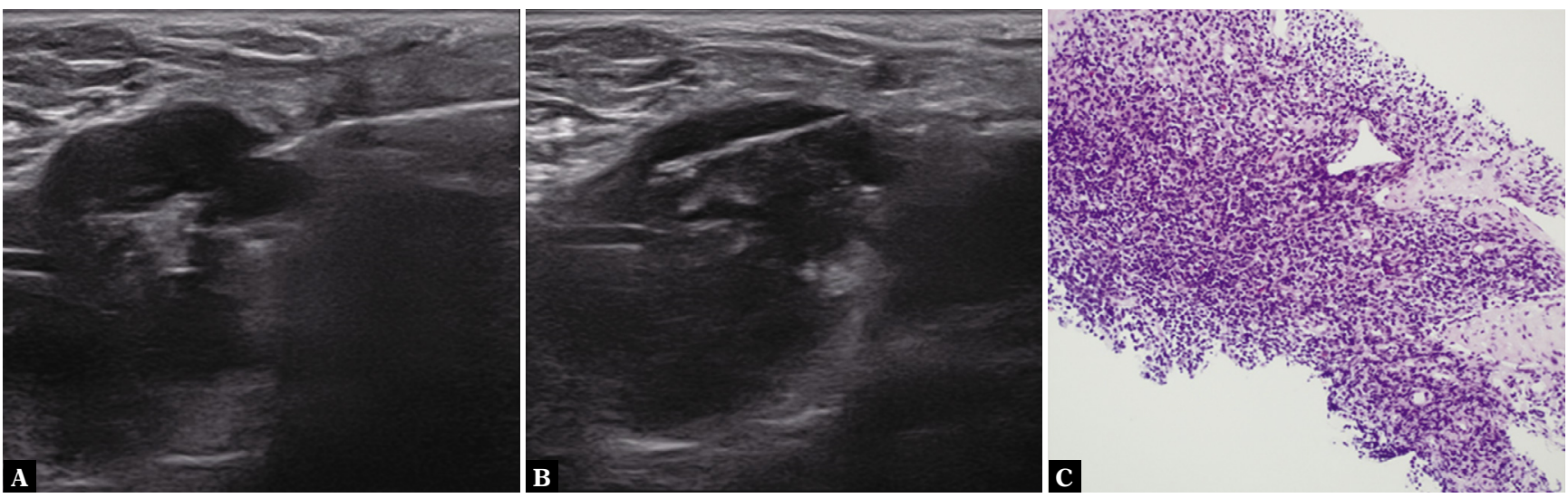

Fig. 1. Example of vaccine-associated adenopathy in a 66-year-old female patient undergoing breast ultrasound for routine breast imaging with history of breast cancer in the left breast. A. US in the left shoulder showing left axillary adenopathy (22 $\mathrm{mm})$ with fatty hilum and thickened cortex. B. A core biopsy was performed. C. The histology was negative for lymph node malignancy (normal lymphocytes and histiocytes in hematoxylin-eosin staining)
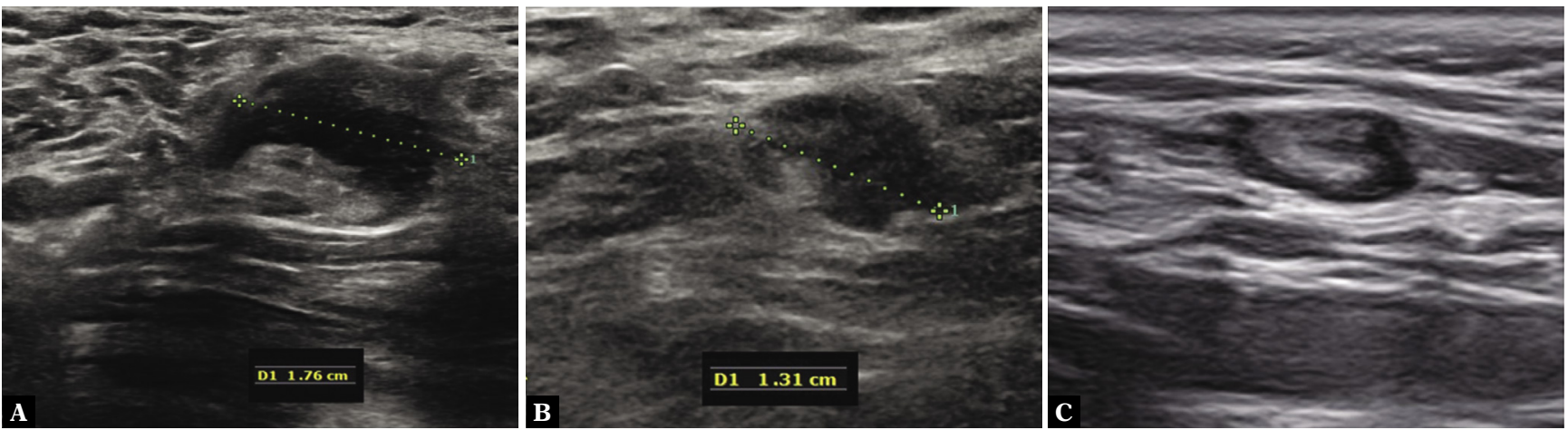

Fig. 2. Example of vaccine-associated adenopathy in a 29-year-old female undergoing breast ultrasound for routine breast imaging due to increased breast density. A. US in the left shoulder showing asymmetric left axillary adenopathy $(18 \mathrm{~mm})$ with preserved fatty hilum but irregular cortex. B. Four-week follow-up: axillary sonography of the same patient demonstrates decreased size (13 cm) and some residual cortical thickening. C. Eight-week follow-up: axillary ultrasound shows normal sized lymph node

few days. However, palpable lymphadenopathy commonly involving the axilla as an immune response to vaccination may also be present ${ }^{(11)}$.

In routine breast imaging, ultrasound is normally performed following screening examination in women with dense breasts $^{(12)}$. In our study, asymptomatic and symptomatic patients presented heterogeneously dense breasts (breast density $\mathrm{C}$ or D in accordance with BIRADS density assessment) and underwent standard breast ultrasound with axillary evaluation, as normally done in our clinical practice ${ }^{(13-14)}$. Ultrasound detection of unilateral axillary adenopathy is possible, and the differential diagnosis is broad, including inflammation, infection or trauma of the breast, thoracic wall or arm, and occult malignancy in a significant portion of patients ${ }^{(15)}$. Classical radiological signs of a normal sized lymph node include: short axis (less than $10 \mathrm{~mm}$ ) with a thin cortex of less than $3 \mathrm{~mm}$. A normal node has an oval shape. Its cortex is thin and of uniform thickness. The cortex is hypoechoic. The contours are well delineated. The hilum, consisting mostly of fat, is wide. The hilar fat is usually hyperechoic but can also take on a non-suspicious hypoechoic aspect. Doppler analysis shows vascularization in the hilum exclusively ${ }^{(16)}$. A pathologic lymph node presents morphological changes of the cortex, the hilum, and the shape: cortex thickening of $>3 \mathrm{~mm}$, presence of focal bulges and peripheral vascularization with the color Doppler, round shape, and hypoechoic aspect of the node ${ }^{(16)}$. Malignancy remains the most serious differential for unilateral axillary adenopathy, but also benign etiologies should be considered, such as local inflammation, infection or trauma. Recognizing this association is crucial in patients with cancer, as failure to do so can lead to under- or overdiagnosis and under- or overtreatment as well as heightened anxiety. This is especially relevant for patients with certain cancers such as breast cancer, head and neck cancers, lymphoma, and melanoma of the back and upper extremities, which have a predilection for metastasizing to these lymph node stations. Adenopathy related to recent vaccination status is not an uncommon benign etiology and has been documented following smallpox, Bacille Calmette-Guerin (BCG), and anthrax vaccination, and HPV vaccine ${ }^{(17)}$. Özütemiz et al. pointed out that no data is actually available regarding the duration of radiologically evident lymphadenopathy or appropriate follow-up intervals ${ }^{(18)}$. Becker et al. recently reported that the Society of Breast Imaging (SBI) was the first imaging society to propose guidelines about axillary lymphadenopathy valuated on imaging. SBI suggested recording personal COVID-19 vaccination history of patients (vaccination dates, injection site, and vaccine type) and highlighted that patients might need to 
consider scheduling screening exams prior to the first dose of their COVID-19 vaccination or 4-6 weeks following the second dose of a COVID-19 vaccine to mitigate the diagnostic dilemma of vaccine-induced lymphadenopathy. For incidentally detected unilateral lymphadenopathy on imaging, a short-term followup in 4-12 weeks after the second vaccine dose is suggested. If lymphadenopathy persists, a biopsy is required to exclude malignancy ${ }^{(19)}$. In our experience, we suggested that patients should return for a follow-up targeted ultrasound scan of the axilla after 4-8 weeks to ensure resolution; indeed, we observed regression of enlarged lymph nodes in five patients with no history of malignancy. Recognition of the association between COVID-19 vaccines administered intramuscularly into the deltoid muscle and adenopathy is important in patients with cancer, as it can lead to under- or overdiagnosis and under- or overtreatment as well as heightened anxiety. This is especially relevant for patients with breast cancer, head and neck cancers, lymphoma, and melanoma, which have a predilection for metastasizing to these lymph node stations. In our series, five patients with a personal history of breast cancer underwent US-guided biopsy with a negative histological results. To reduce diagnostic concerns, SBI proposes to administer COVID-19 vaccine "on the side contralateral to the primary cancer" in oncologic patients to reduce confounding findings, and in cases involving post-vaccination lymphadenopathy, a short-term imaging follow-up with ultrasound and- or tissue diagnosis can be considered ${ }^{(19)}$. With more available clinical data, patient management will be guided by the clinical context, particularly in patients with cancer history, in order to reduce false-positive, unnecessary additional imaging, and biopsies throughout the COVID-19 vaccination period.

\section{References}

1. Grasselli G, Pesenti A, Cecconi M: Critical care utilization for the COVID-19 outbreak in Lombardy, Italy: early experience and forecast during an emergency response. JAMA 2020; 323: 1545-1546.

2. Fleming TR, Nason M, Krause PR, Longini IM, Henao-Restrepo AM: COVID-19 vaccine trials: the potential for "hybrid” analyses. Clin Trials 2021; 18: 391-397.

3. Gringeri M, Mosini G, Battini V, Cammarata G, Guarnieri G, Carnovale C et al.: Preliminary evidence on the safety profile of BNT162b2 (Comirnaty): new insights from data analysis in EudraVigilance and adverse reaction reports from an Italian health facility. Hum Vaccin Immunother 2021; 17: 2969-2971.

4. Sahin U, Muik A, Vogler I, Derhovanessian E, Kranz LM, Vormehr M et al.: BNT162b2 vaccine induces neutralizing antibodies and polyspecific T cells in humans. Nature 2021; 595: 572-577.

5. Skowronski DM, De Serres G: Safety and efficacy of the BNT162b2 mRNA Covid-19 Vaccine. N Engl J Med 2021; 384: 1576-1577.

6. Cohen D, Hazut Krauthammer S, Cohen YC, Perry C, Avivi I, Herishanu Y et al.: Correlation between BNT162b2 mRNA Covid-19 vaccine-associated hypermetabolic lymphadenopathy and humoral immunity in patients with hematologic malignancy. Eur J Nucl Med Mol Imaging 2021; 48: 3540-3549.

7. Cohen D, Krauthammer SH, Wolf I, Even-Sapir E: Hypermetabolic lymphadenopathy following administration of BNT162b2 mRNA Covid-19 vaccine: incidence assessed by $\left[{ }^{18}\right.$ F]FDG PET-CT and relevance to study interpretation. Eur J Nucl Med Mol Imaging 2021; 48: 1854-1863.

8. Eifer M, Tau N, Alhoubani Y, Kanana N, Domachevsky L, Shams J et al.: Covid-19 mRNA vaccination: age and immune status and its association with axillary lymph node PET/CT uptake. J Nucl Med 2021.

9. Hiller N, Goldberg SN, Cohen-Cymberknoh M, Vainstein V, Simanovsky $\mathrm{N}$ : Lymphadenopathy associated with the COVID-19 vaccine. Cureus 2021; 13: e13524.

\section{Conclusion}

COVID-19 vaccination in the deltoid muscle may be associated with clinically palpable benign ipsilateral axillary lymphadenopathy. Imaging information and recommendations are necessary to help take more informed and effective care of patients in order to prevent unnecessary changes in therapeutic management, reduce patients' emotional stress or avoid a redundant biopsy.

\section{Ethics approval and consent to participate}

Our study is included in IRB approved clinical trials (NCT03033030, NCT02066142 and NCT04253366; CER 262/2019; 514/2014) with ethical approval and consent to participate.

\section{Consent for publication}

Patients signed an informed consent to publish.

\section{Availability of data and material}

The datasets used and/or analyzed during the current study are available from the corresponding author on reasonable request.

\section{Conflict of interest}

Authors do not report any financial or personal connections with other persons or organizations, which might negatively affect the contents of this publication and/or claim authorship rights to this publication.

10. Keshavarz P, Yazdanpanah F, Rafiee F, Mizandari M: Lymphadenopathy following COVID-19 vaccination: imaging findings review. Acad Radiol 2021; 28: 058-1071.

11. Tu W, Gierada DS, Joe BN: COVID-19 vaccination-related lymphadenopathy: what to be aware if. Radiol Imaging Cancer 2021; 3: e210038.

12. Vourtsis A, Berg WA: Breast density implications and supplemental screening. Eur Radiol 2019; 29: 1762-1777.

13. Tagliafico AS, Mariscotti G, Valdora F, Durando M, Nori J, La Forgia D et al:: A prospective comparative trial of adjunct screening with tomosynthesis or ultrasound in women with mammography-negative dense breasts (ASTOUND-2). Eur J Cancer 2018; 104: 39-46.

14. Tagliafico AS, Valdora F, Mariscotti G, Durando M, Nori J, La Forgia D et al.: An exploratory radiomics analysis on digital breast tomosynthesis in women with mammographically negative dense breasts. Breast 2018; 40: 92-96.

15. Dialani V, James DF, Slanetz PJ: A practical approach to imaging the axilla. Insights Imaging 2015; 6: 217-229.

16. Maxwell F, de Margerie Mellon C, Bricout M, Cauderlier E, Chapelier M, Albiter M et al.: Diagnostic strategy for the assessment of axillary lymph node status in breast cancer. Diagn Interv Imaging 2015; 96: 1089-1101.

17. Patel T, Given-Wilson RM, Thomas V: The clinical importance of axillary lymphadenopathy detected on screening mammography: revisited. Clinical radiology. 2005; 60: 64-71.

18. Özütemiz C, Krystosek LA, Church AL, Chauhan A, Ellermann JM, Domingo-Musibay E et al.: Lymphadenopathy in COVID-19 vaccine recipients: diagnostic dilemma in oncology patients. Radiology 2021; 300: E296-E300.

19. Becker AS, Perez-Johnston R, Chikarmane SA, Chen MM, El Homsi M, Feigin $\mathrm{KN}$ et al.: Multidisciplinary recommendations regarding postvaccine adenopathy and radiologic imaging: Radiology Scientific Expert Panel. Radiology 2021; 300: E323-E327. 